\title{
A Fast-Decodable Code Structure for Linear Dispersion Codes
}

\author{
X. G. Dai, Graduate Student Member, IEEE, S. W. Cheung, Senior Member, IEEE, and T. I. Yuk, Member, IEEE
}

\begin{abstract}
This paper proposes the design of a new family of fast-decodable, full-rank, flexible-rate linear dispersion codes (LDCs) for MIMO systems with arbitrary numbers of transmit and receive antennas. The codewords of LDCs can be expressed as a linear combination of certain dispersion matrices and, in this new family of LDCs, we propose to have orthogonal rows in as many dispersion matrices as possible. We show that, with the proposed code, the number of levels in the tree search and hence the complexity of the sphere decoder (SD) at the receiver can be substantially reduced. Monte Carlo computer simulation has shown that the LDCs with and without the orthogonal structure have nearly identical bit-error-rate (BER) performances. However, the complexity of the SD used for decoding the proposed family of LDCs is substantially reduced.
\end{abstract}

Index Terms-Linear dispersion code, complexity of sphere decoding, MIMO system, fast-decodable code.

\section{INTRODUCTION}

$\mathbf{T}$ HE complexity of the decoding/detection process for space-time (ST) codes is a major constraint for the implementation of high speed Multiple-Input-Multiple-Output (MIMO) systems over wireless channels. It is well-known that Maximum likelihood (ML) decoding can achieve the minimum block-error rate (BLER) performance of ST codes. An ML decoder requires joint detection of an entire block of symbols, so the complexity increases exponentially with the number of transmitted symbols in a codeword, making it very difficult to use for large codeword sizes.

In 1998, Alamouti proposed a remarkable ST code which could achieve the minimum BLER by using a decoder with much less complexity for MIMO systems with two transmit antennas [1]. Subsequently, orthogonal space-time block codes (OSTBCs) which allow the use of low-complexity ML decoders were introduced [2], but such OSTBCs cannot achieve the full transmission rates for MIMO systems using more than two transmit antennas [3]. Then quasi-orthogonal STBCs (QOSTBCs) were proposed to achieve the full transmission rates at the price of lower diversity gain [4]. Recently, rateone QOSTBCs have been studied to provide full diversity gain with low decoding complexities [5], [6], but the transmission rate is limited to just one symbol/time interval.

Sphere Decoding (SD) has attracted much attention because of its ability to substantially reduce the computational complexity of the ML decoder, yet having the same errorrate performance [7]. SD is a search-based algorithm and performs a lattice point search for each received symbol

Manuscript received June 26, 2008; revised December 1, 2008 and February 23, 2009; accepted June 11, 2009. The associate editor coordinating the review of this letter and approving it for publication was M. Saquib.

The authors are with the Department of Electrical and Electronics Engineering, The University of Hong Kong (e-mail: \{swcheung, xgdai, tiyuk\}@eee.hku.hk).

Digital Object Identifier 10.1109/TWC.2009.080841 block. The complexity of the searching process increases exponentially with the number of symbols in a block [8]. To speed up the searching process, different searching strategies have been proposed to implement the SD algorithms [7], [9] and modifications of these SD algorithms have also been made to reduce the decoding complexity [10].

Linear dispersion codes (LDCs) were first proposed in [11]. By combining the advantages of OSTBCs and SD, a family of fast decodable full-rate, full-rank linear dispersion codes (LDCs) for $2 \times 2$ MIMO system was constructed in [12]. With this family of LDCs, the decoding operation was much simplified. Biglieri, Hong and Viterbo extended the concept of this fast-decodable space-time block code (STBC) design to a $4 \times 2$ MIMO system and presented a family of quasiorthogonal structured codes, achieving a reduction of 4 levels in the tree search for SD [13]. Similar ideas were also used in [14], [15] where the STBCs were called the multi-strata space-time code and silver code as a cyclic division algebra. However, these codes were designed only for $2 \times 2 \mathrm{MIMO}$ systems.

Motivated by the above work, we propose a new family of fast-decodable, full-rank LDCs with flexible transmission rates for MIMO systems. In LDCs, each codeword can be expressed as a linear combination of certain dispersion matrices [11]. In this new family of LDCs, we propose to have orthogonal rows in as many dispersion matrices as possible in the LDCs. We show that, for LDCs with this structure, the number of levels required in the search trees for SD can be substantially reduced and the reduction depends on the number of transmitted symbols in a codeword and also the modulation schemes used. The major advantages of this new family of LDCs are that it can be designed for MIMO systems with arbitrary numbers of transmit and receive antennas and it can support flexible transmission rate.

The remainder of this paper is organized as follows. The system model considered herein is defined in Section II. In Section III, we present the design and construction of a new family of fast-decodable LDCs and describe the corresponding simplified SD algorithm. In Section IV, computer simulation results and discussions of our proposed code are presented. Section $\mathrm{V}$ is the conclusions.

\section{SySTEM MODEL}

Consider an $N_{t} \times N_{r}$ MIMO system having $N_{t}$ transmit antennas and $N_{r}$ receive antennas, over a block-fading channel. Assuming the block length of each codeword is $T$ (which is the number of time intervals to transmit a complete coded symbol block), the $N_{r} \times T$ received signal matrix $\mathbf{R}$ is given by:

$$
\mathbf{R}=\mathbf{H C}+\mathbf{W}
$$


where $\mathbf{H} \in \mathcal{C}^{N_{r} \times N_{t}}$ is the channel matrix which is assumed to be constant during a codeword interval and known to the receiver but unknown to the transmitter, $\mathbf{W} \in \mathcal{C}^{N_{r} \times T}$ is the complex additive white Gaussian noise (AWGN) matrix with elements being independently and identically distributed (iid) and following the normal distribution $N_{C}\left(0, N_{0}\right)$, and $\mathbf{C} \in$ $\mathcal{C}^{N_{t} \times T}$ is the codeword matrix of a LDC. The codeword matrix C can be expressed as [11]:

$$
\mathbf{C}=\sum_{i=1}^{N} \mathbf{M}_{i} \cdot s_{i}
$$

where $\left\{\mathbf{M}_{i}\right\}_{i=1}^{N}$ are the complex dispersion matrices of the LDC, $\left\{s_{i}\right\}_{i=1}^{N}$ are the transmitted symbols taking values from some complex constellation in a finite set $\mathcal{S}$, and $N$ is the number of symbols in one codeword. The transmitted symbols $\left\{s_{i}\right\}_{i=1}^{N}$ in a codeword can be expressed in vector form as $\mathbf{s}=\left[s_{1}, s_{2}, s_{3}, \ldots, s_{N}\right] \in \mathcal{C}^{N}$. All elements in $\mathbf{R}, \mathbf{H}, \mathbf{C}$ and $\mathbf{W}$ of (1) are complex variables.

The vectorization of a matrix $\mathbf{A}$, denoted by vec $(\mathbf{A})$, converts the matrix into a single column vector by stacking successive columns of the matrix. Substituting (2) into (1) and taking vectorization on both sides of the resultant equation give:

$$
\overline{\mathbf{r}}=\mathbf{K} \overline{\mathbf{H}} \overline{\mathbf{s}}+\overline{\mathbf{w}}
$$

where $\quad \overline{\mathbf{r}} \quad=\quad \operatorname{vec}(\mathbf{R})$

$\left[r_{1,1}, r_{2,1}, \ldots, r_{N_{r}, 1}, r_{1,2}, \ldots, r_{N_{r}, 2}, \ldots, r_{N_{r}, T}\right] \quad$ with $r_{i, j}$ being the entry in the $i$ th row and $j$ th column of matrix $\mathbf{R}$ and []$^{T}$ denoting matrix transposition, $\overline{\mathbf{X}}=\left[\operatorname{vec}\left(\mathbf{M}_{0}\right), \operatorname{vec}\left(\mathbf{M}_{1}\right), \ldots, \operatorname{vec}\left(\mathbf{M}_{N}\right)\right]$, $\overline{\mathbf{s}}=\operatorname{vec}(\mathbf{s})=\mathbf{s}^{T}=\left[s_{1}, \ldots, s_{N}\right]^{T}$ and $\overline{\mathbf{w}}=\operatorname{vec}(\mathbf{W})$. In (3), $\mathbf{K}$ is a stacked channel matrix defined as $\mathbf{K}=\mathbf{I} \otimes \mathbf{H}$, where $\mathbf{K} \in \mathcal{C}^{N_{r} T \times N_{t} T}, \mathbf{I}$ is a $T \times T$ identity matrix and $\otimes$ denotes the Kronecker product. Separating the real and imaginary parts of the elements in $\overline{\mathbf{r}}, \overline{\mathbf{s}}$ and $\overline{\mathbf{w}}$ of (3) and then vectorizing them give the real-valued expression:

$$
\tilde{\mathbf{r}}=\tilde{\mathbf{G}} \tilde{\mathbf{s}}+\tilde{\mathbf{w}}
$$

where $\tilde{\mathbf{r}}=\left[\Re\left(\overline{\mathbf{r}}^{T}\right), \Im\left(\overline{\mathbf{r}}^{T}\right)\right]^{T}, \tilde{\mathbf{s}}=[\Re(\mathbf{s}), \Im(\mathbf{s})]^{T}=$ $\left[\Re\left(s_{1}\right), \ldots, \Re\left(s_{N}\right), \Im\left(s_{1}\right), \ldots, \Im\left(s_{N}\right)\right]^{T}, \quad$ and $\tilde{\mathbf{w}}=$ $\left[\Re\left(\overline{\mathbf{w}}^{T}\right), \Im\left(\overline{\mathbf{w}}^{T}\right)\right]^{T}$, with $\Re($.$) and \Im($.$) denoting the real and$ imaginary parts, respectively. In (4), $\tilde{\mathbf{G}}$ is a $2 N_{r} T \times 2 N$ real matrix given by:

$$
\widetilde{\mathbf{G}}=\left[\begin{array}{cc}
\Re(\mathbf{K} \overline{\mathbf{X}}) & -\Im(\mathbf{K} \overline{\mathbf{X}}) \\
\Im(\mathbf{K} \overline{\mathbf{X}}) & \Re(\mathbf{K} \overline{\mathbf{X}})
\end{array}\right]
$$

Here, we adopt an ordering scheme similar to the one used in [16] to construct our proposed code structure. First, we arrange the vector $\tilde{\mathbf{s}}$ in (4) to

$$
\begin{aligned}
\tilde{\tilde{\mathbf{s}}} & =\left[\Re\left(s_{1}\right), \Im\left(s_{1}\right), \Re\left(s_{2}\right), \Im\left(s_{2}\right), \ldots, \Re\left(s_{N}\right), \Im\left(s_{N}\right)\right]^{T} \\
& =\left[\tilde{\tilde{s}}_{1}, \tilde{\tilde{s}}_{2}, \tilde{\tilde{s}}_{3}, \ldots, \tilde{\tilde{s}}_{N}\right]^{T}
\end{aligned}
$$

To keep the received signal vector $\tilde{\mathbf{r}}$ in (4) unchanged, we need to arrange the columns of $\tilde{\mathbf{G}}$ correspondingly. That is, for $\tilde{\mathbf{G}}=\left[\tilde{\mathbf{g}}_{1}, \tilde{\mathbf{g}}_{2}, \ldots, \tilde{\mathbf{g}}_{2 N}\right]$, where $\tilde{\mathbf{g}}_{i}$, for $\mathrm{i}=1,2, \ldots, 2 N$, denotes the $i$ th column of $\tilde{\mathbf{G}}$, we arrange the columns of $\tilde{\mathbf{G}}$ to give:

$$
\tilde{\tilde{\mathbf{G}}}=\left[\tilde{\mathbf{g}}_{1}, \tilde{\mathbf{g}}_{N+1}, \tilde{\mathbf{g}}_{2}, \tilde{\mathbf{g}}_{N+2}, \ldots \tilde{\mathbf{g}}_{N}, \tilde{\mathbf{g}}_{2 N},\right]
$$

With these arrangements, the received signal vector $\tilde{\mathbf{r}}$ in (4) can be re-written as:

$$
\tilde{\mathbf{r}}=\tilde{\tilde{\mathbf{G}}} \tilde{\tilde{\mathbf{s}}}+\tilde{\mathbf{w}}
$$

\section{PRoposed FAST-DECODABLE CODE}

\section{A. Proposed LDC code structure}

Here we present a new family of LDCs having an orthogonal row structure that enables fast sphere decoding. In this new type of LDC, the first $m$ dispersion matrices among the $N$ dispersion matrices $\left\{\mathbf{M}_{i}\right\}_{i=1}^{N}$ in (2) satisfy the following condition:

$$
\mathbf{M}_{i} \mathbf{M}_{j}^{H}=\mathbf{0}(i \neq j ; i, j \leq m)
$$

where $\mathbf{0}$ is an $N_{t} \times N_{t}$ matrix with all elements being zero and (.) ${ }^{H}$ denotes the transpose conjugate of a matrix (.). This means that among the first $m$ dispersion matrices, the rows of one dispersion matrix are orthogonal to the rows of any other dispersion matrix.

\section{B. Simplified sphere decoding for proposed LDC}

Sphere decoding attempts to obtain the solution of [7]:

$$
\hat{\mathbf{s}}=\arg \min _{\tilde{\mathbf{s}} \in \mathcal{S}}\|\tilde{\mathbf{r}}-\tilde{\tilde{\mathbf{G}}} \tilde{\tilde{\mathbf{s}}}\|^{2}
$$

To do this, we can first conduct the QR decomposition on $\tilde{\tilde{G}}$ :

$$
\tilde{\tilde{\mathbf{G}}}=\mathbf{Q}\left[\begin{array}{c}
\mathbf{P} \\
\mathbf{0}_{\left(2 N_{r} T-2 N\right) \times 2 N}
\end{array}\right]
$$

where $\mathbf{P} \in \mathcal{R}^{2 N \times 2 N}$ is an upper triangular matrix, $\mathbf{Q} \in$ $\mathcal{R}^{2 N_{r} T \times 2 N_{r} T}$ is an orthonormal matrix, and $N_{r} T \geq N$. Then (10) can be written as:

$$
\hat{\mathbf{s}}=\arg \min _{\tilde{\tilde{\mathbf{s}}} \in \mathcal{S}}\|\mathbf{y}-\mathbf{P} \tilde{\tilde{\mathbf{s}}}\|^{2}
$$

where $\mathbf{y}=\mathbf{Q}_{1}^{T} \tilde{\mathbf{r}}, \mathbf{Q}_{1}$ is the first $2 N$ orthonormal columns of Q. Based on (12), the SD can now search only the points within a hypersphere centered at the received signal with radius $d$ by solving the following inequality iteratively [7], [9], [10]:

$$
\|\mathbf{y}-\mathbf{P} \tilde{\tilde{\mathbf{s}}}\|^{2} \leq d^{2}
$$

There are many papers discussing how to choose the initial radius $d_{0}$ of the hypersphere [7], [9], [10]. Since the initial setting of $d_{0}$ does not affect the advantage of using our proposed algorithm, the issue of choosing $d_{0}$ is not discussed here. In our studies, we simply set $d_{0}$ to be the noise variance of the channel to initialize the searching process.

To explain how the orthogonal row structure can be used to simplify the SD process, we first introduce the following Lemma:

Lemma 1: If a LDC has an orthogonal row structure and so satisfies (9), the elements $p_{i, j}$, for $i=1,2, \ldots, 2 m-1$ and $j=$ $i+1, i+2, \ldots, 2 m$, in the upper triangular matrix $\mathbf{P}$ in (12) are all zeros. The proof is omitted here due to the page limit.

According to Lemma 1, if a LDC with an orthogonal row structure is used, there will be a cluster of zeros in the upper triangular matrix $\mathbf{P}$ in (12). In (13), there are a total of $2 N$ inequalities, corresponding to the $2 N$ rows in the matrix 
equation. To solve the inequality iteratively, the SD starts from the bottom row of the matrix and works upwards to the first row. When the SD has completed the $(2 m+1)$ th row, the values of $\tilde{\tilde{s}}_{2 N}, \tilde{\tilde{s}}_{2 N-1}, \ldots, \tilde{\tilde{s}}_{2 m+1}$ will have been determined. Then benefiting from the cluster of zeros in $\mathbf{P}$, we do not need to use the values of $\tilde{\tilde{s}}_{i+1}, \tilde{\tilde{s}}_{i+2}, \ldots, \tilde{\tilde{s}}_{2 m}$ to determine the values of $\left\{\tilde{\tilde{s}}_{i}\right\}$ for $i=1$ to $2 m$. Instead, we can simply determine the value of $\tilde{\tilde{s}}_{i}$ by hard decoding:

$$
\tilde{\tilde{s}}_{i}=\left\lceil\left(y_{i}-\sum_{k=2 m+1}^{2 N} p_{i, k} \tilde{\tilde{s}}_{k}\right) / p_{i, i}\right\rfloor
$$

where $\lceil a\rfloor$ means the possible value of $\tilde{\tilde{s}}_{i}$ closest to $a$. Thus our proposed code structure can reduce the tree search by $2 m$ levels in SD. Note that compared to the complexity of tree search, the complexity of hard decoding can be neglected. Here, it can be seen that our method can be applied to other SD algorithms such as in [9], [10].

\section{Construction of LDCs with Orthogonal Row Structure}

One simple method to design the dispersion matrices $\left\{\mathbf{M}_{i}\right\}_{i=1}^{N}$ to have the orthogonal row structure is to find $m \cdot N_{t}$ orthogonal vectors with length $T$ and use them as the row vectors in the $m$ dispersion matrices. In the vector space of $T$ dimensions, there are at most $T$ orthogonal vectors. LDC designed with this method will therefore have the constraint of $T \geq m \cdot N_{t}$. As all of the dispersion matrices should have the same average power, in the design, we impose the following power constraint [11]:

$$
\operatorname{tr}\left\{\mathbf{M}_{n} \mathbf{M}_{n}^{H}\right\}=\frac{T}{N}, n=1, \ldots, N
$$

where $\operatorname{tr}\{$.$\} denotes the trace of a matrix.$

To ensure that the LDC is capacity-optimal, the vectorized coding matrix $\overline{\mathbf{X}}$ in (3) must also satisfy the following condition [11]:

$$
\overline{\mathbf{X}}^{H} \overline{\mathbf{X}}=\frac{T}{N} \mathbf{I}_{N}
$$

which means that the columns of $\overline{\mathbf{X}}$ should be orthogonal to each others. It can easily be shown that, if the first $m$ dispersion matrices satisfy (9), the first $m$ columns of $\overline{\mathbf{X}}$, $\operatorname{vec}\left(\mathbf{M}_{1}\right), \ldots, \operatorname{vec}\left(\mathbf{M}_{m}\right)$, must always be orthogonal to each others. Thus, for the LDC to satisfy (16), we only need to normalize these matrices $\left\{\mathbf{M}_{i}\right\}_{i=1}^{m}$ so that their Frobenius norms are $\sqrt{\frac{T}{N}}$. Finally, the Gram-Schmidt method is used to generate the remaining $N-m$ dispersion matrices to satisfy (16). The LDC constructed in this way always will satisfy the power constraint and is capacity-optimal.

\section{Simulation Results and Discussions}

Studies have been carried out on LDCs with our proposed fast-decodable code structure using four different MIMO systems: two $2 \times 4$ MIMO systems transmitting 4 QPSK or 16QAM symbols in a codeword with a length of 4 (i.e., $N_{t}=2, N_{r}=4, T=4$ and $N=4$ ) and two $3 \times 3$ MIMO systems transmitting 6 QPSK or 16QAM symbols in a codeword with a length of 6 (i.e., $N_{t}=3, N_{r}=3, T=6$ and $N=6$ ). The LDCs, with and without the orthogonal
TABLE I

MINIMUM DETERMINANTS OF THE CODEWORD DIFFERENCE MATRICES OF LDCS WITH AND WITHOUT OUR STRUCTURE IN FOUR SYSTEMS.

\begin{tabular}{|l|l|l|l|l|}
\hline & $\begin{array}{l}2 \times 4 \\
\text { QPSK } \\
\mathrm{N}=4\end{array}$ & $\begin{array}{l}2 \times 4 \\
16 \mathrm{QAM} \\
\mathrm{N}=4\end{array}$ & $\begin{array}{l}3 \times 3 \\
\text { QPSK } \\
\mathrm{N}=6\end{array}$ & $\begin{array}{l}3 \times 3 \\
\text { 16QAM } \\
\mathrm{N}=6\end{array}$ \\
\hline $\begin{array}{l}\text { With our } \\
\text { structure }\end{array}$ & 0.9950 & 0.0400 & 0.2358 & 0.0019 \\
\hline $\begin{array}{l}\text { Without our } \\
\text { structure }\end{array}$ & 0.9606 & 0.0389 & 0.2096 & 0.0017 \\
\hline
\end{tabular}

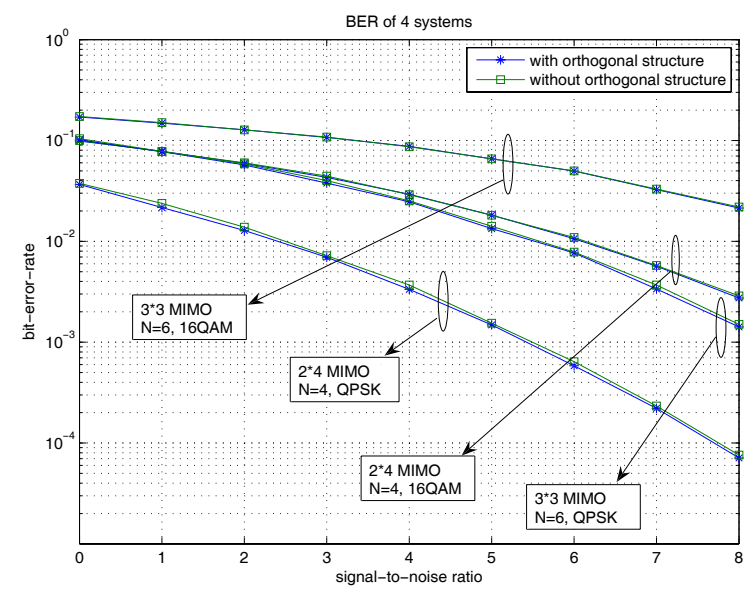

Fig. 1. BER performances of LDCs in MIMO systems studied.

row structure, used for study in these systems were obtained using random-search optimization (for one million times) with the rank and determinant criteria. The minimum determinants of the codeword difference matrices obtained for the LDCs with and without our code structure are shown in Table 1. It can be seen that our proposed LDCs have larger minimum determinants values. The reason is that since the orthogonal row structure makes the rows of the first $m$ dispersion matrices orthogonal to each others, the first $m$ dispersion matrices are guaranteed to be full-rank with relatively large determinants. When this constraint is used as the starting point in a random search with a finite number of searches, the final LD code obtained is likely to have a relatively larger determinant than that of the final LD code obtained using a random search with the same number of searches in the set of LD codes without the constraint. Monte Carlo simulation has been used to evaluate the BER performances of these LDCs and results are shown in Fig. 1. It can be seen that, in each of these four systems, the BER performances of the LDCs, with and without the orthogonal row structure, are nearly identical, indicating that the orthogonal row structure in the LDC does not degrade the BER performance.

As mentioned in [8], the complexity of SD can be measured by the number of visited nodes in the SD tree search process. The Monte Carlo simulation results on the average number of visited nodes in the conventional SD and our simplified SD for the $2 \times 4$ and $3 \times 3 \mathrm{MIMO}$ systems, transmitting 4 and 6 QPSK symbols in a codeword, are shown in Fig. 2. It can be seen that in the $2 \times 4$ MIMO system transmitting 4 QPSK 


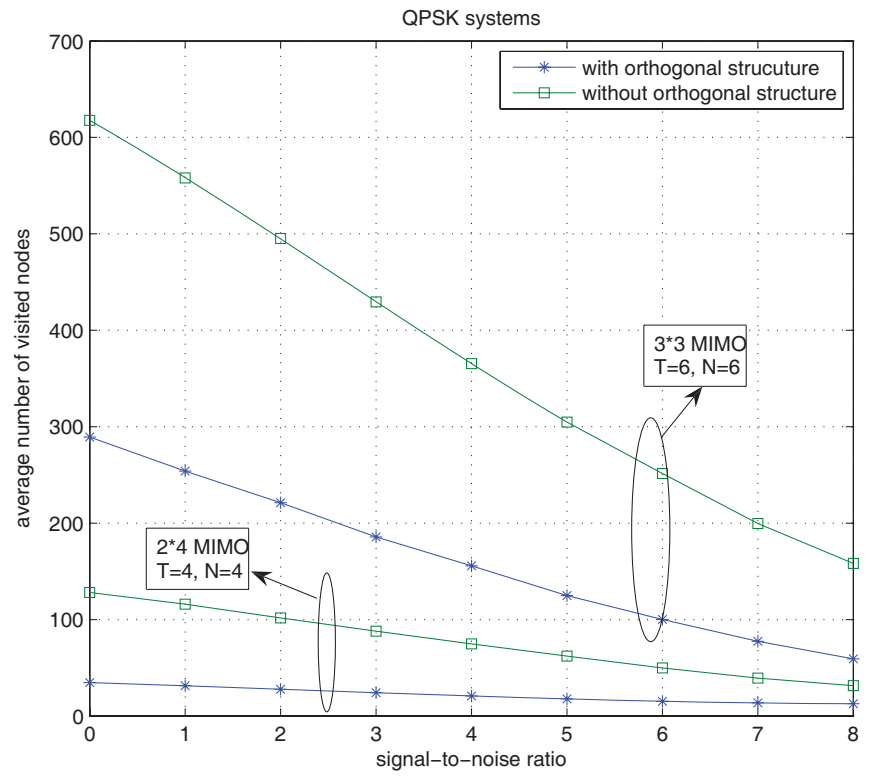

Fig. 2. Average number of visited nodes in SDs of $2 \times 4$ and $3 \times 3$ MIMO systems transmitting QPSK.

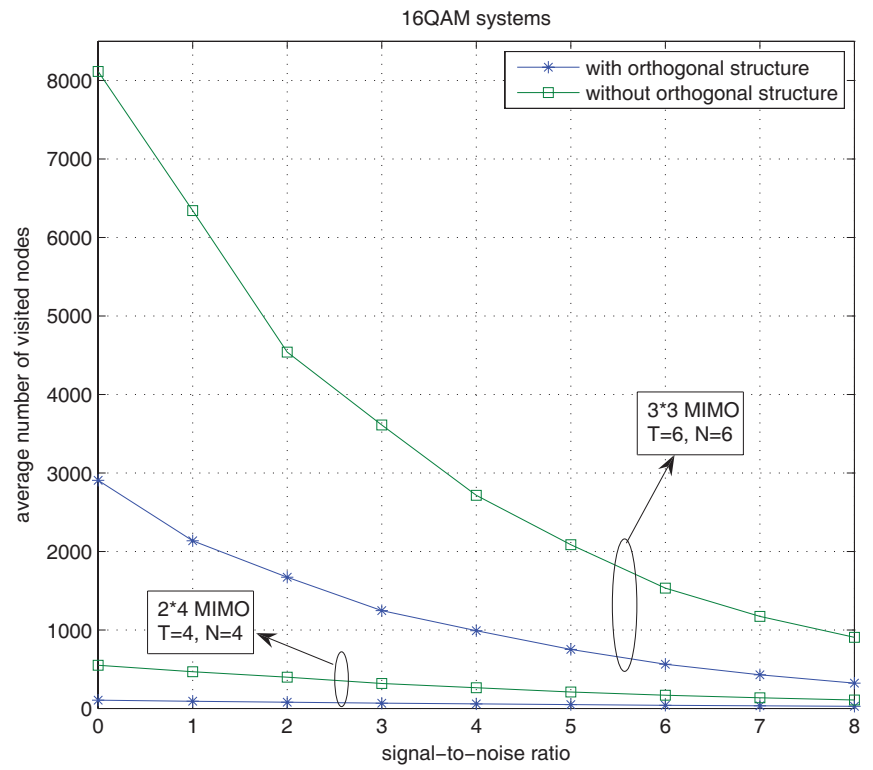

Fig. 3. Average number of visited nodes in SDs of $2 \times 4$ and $3 \times 3$ MIMO transmitting 16QAM.

symbols, our simplified SD reduces the complexity by about 59-73\%. In the $3 \times 3$ MIMO system transmitting 6 QPSK symbols, it reduces the complexity by about $53-63 \%$. Monte Carlo simulations have also been carried out on the two MIMO systems using 16QAM symbols and the results are shown in Fig. 3. In the $2 \times 4$ MIMO system transmitting 4 16QAM symbols in a codeword, the complexity is reduced by about $75-81 \%$ using our simplified SD. While in the $3 \times 3$ MIMO system transmitting 6 16QAM symbols in a codeword, the complexity reduction is about $63-67 \%$. Thus the reduction is more significant for signals with higher levels of modulation.

\section{CONCLUSION}

The design of a new family of fast-decodable, full-rank, flexible-rate LDCs with orthogonal row structure has been proposed and presented to reduce the complexity of SD used in the MIMO systems. Monte Carlo simulation results have shown that the LDCs, with and without the orthogonal row structure, have nearly identical BER performances. However, the complexity of the SD can be substantially reduced by using LDC with orthogonal row structure and the reduction is more significant for the LDCs with higher-level modulations.

\section{REFERENCES}

[1] S. M. Alamouti, "A simple transmit diversity technique for wireless communications," IEEE J. Select. Areas Commun., vol. 16, pp. 14511458, Oct. 1998.

[2] V. Tarokh, N. Seshadri, and A. R. Calderbank, "Space-time codes for high data rate wireless communication: performance criteria in the presence of channel estimation errors, mobility, and multiple paths," IEEE Trans. Commun., vol. 47, pp. 199-207, Feb. 1999.

[3] O. Tirkkonen and A. Hottinen, "Square-matrix embeddable space-time block codes for complex signal constellations," IEEE Trans. Inform. Theory, vol. 48, pp. 384-395, Feb. 2002.

[4] H. Jafarkhani, "A quasi-orthogonal space-time block code," IEEE Commun. Lett., vol. 49, pp. 1-4, Jan. 2001.

[5] L. Xian and H. P. Liu, "Rate-one space-time block codes with full diversity," IEEE Trans. Commun., vol. 53, pp. 1986-1900, Dec. 2005.

[6] D. N. Dao, C. Yuen, C. Tellambura, Y. L. Guan, and T. T. Tjhung, "Fourgroup decodable space-time block codes," IEEE Trans. Signal Processing, vol. 56, pp. 423-430, Jan. 2008.

[7] E. Viterbo and J. Boutros, "A universal lattice code decoder for fading channel," IEEE Trans. Inform. Theory, vol. 45, pp. 1639-1642, July 1999.

[8] J. Jalden and B. Ottersten, "On the complexity of sphere decoding in digital communications," IEEE Trans. Signal Processing, vol. 53, pp. 1474-1484, Apr. 2005.

[9] E. Agrell, T. Eriksson, A. Vardy, and K. Zeger, "Closest point search in lattices," IEEE Trans. Inform. Theory, vol. 48, pp. 2201-2214, Aug. 2002.

[10] M. Damen, H. E. Gamal, and G. Caire, "On maximum-likelihood detection and the search for the closest lattice point," IEEE Trans. Inform. Theory, vol. 49, pp. 2389-2402, Oct. 2003.

[11] B. Hassibi and B. M. Hochwald, "High-rate codes that are linear in space and time," IEEE Trans. Inform. Theory, vol. 48, pp. 1804-1824, July 2002.

[12] J. Paredes, A. Gershman, and M. G. Alkhanari, "A new full-rate full diversity space-time block code with non-vanishing determinants and simplified maximum-likelihood decoding," IEEE Trans. Signal Processing, vol. 56, pp. 2461-2469, June 2008.

[13] E. Biglieri, Y. Hong, and E. Viterbo, "A fast-decodable, quasi-orthogonal space-time block code for $4 \times 2$ MIMO," in Proc. Allerton Conference on Communication, Control and Computing, Sept. 2007, pp. 921-926.

[14] M. Samuel and M. P. Fitz, "Reducing the detection complexity by using $2 \times 2$ multi-strata space-time codes," in Proc. IEEE International Symposium on Information Theory, Nice, France, June 2007, pp. 19461950.

[15] C. Hollanti, J. Lahtonen, K. Ranto, R. Vehkalahti, and E. Viterbo, "On the algebraic structure of the silver code: a $2 \times 2$ perfect space-time block code," in Proc. IEEE Information Theory Workshop, Porto, Portugal, May 2008, pp. 91-94.

[16] L. Azzam and E. Ayanoglu, "Reduced complexity sphere decoding for square QAM via a new lattice representation," in Proc. IEEE Globecom, Washington DC, Nov. 2007, pp. 4242-4246. 